\title{
Combination of modified mixing technique and low frequency ultrasound to control the elution profile of vancomycin-loaded acrylic bone cement
}

\section{A. Wendling, \\ D. Mar, \\ N. Wischmeier, \\ D. Anderson, \\ T. Mclff}

Department of

Orthopedic Surgery,

University of Kansas

Medical Center,

Kansas, United States
A. Wendling, BA, MD Candidate

- D. Mar, MS, PhD Candidate

N. Wischmeier, MD, Orthopedic

Surgery Resident

D. Anderson, MD, Orthopedic

Surgeon

- T. Mclff, PhD, Associate

Professor

Department of Orthopedic

Surgery, University of Kansas

Medical Center, Kansas City,

Kansas, USA

Correspondence should be sent to Dr. T. Mclff; email: tmciff@kumc.

edu

doi: 10.1302/2046-3758.

$52.2000412 \$ 2.00$

Bone Joint Res 2016;5:26-32. Received: 06 March 2015;

Accepted: 01 December 2015

\section{Objectives}

The objective of this study was to determine if combining variations in mixing technique of antibiotic-impregnated polymethylmethacrylate (PMMA) cement with low frequency ultrasound (LFUS) improves antibiotic elution during the initial high phase (Phase I) and subsequent low phase (Phase II) while not diminishing mechanical strength.

\section{Methods}

Three batches of vancomycin-loaded PMMA were prepared with different mixing techniques: a standard technique; a delayed technique; and a control without antibiotic. Daily elution samples were analysed using flow injection analysis (FIA). Beginning in Phase II, samples from each mix group were selected randomly to undergo either five, 15, 45, or 0 minutes of LFUS treatment. Elution amounts between LFUS treatments were analysed. Following Phase II, compression testing was done to quantify strength. A-priori t-tests and univariate ANOVAs were used to compare elution and mechanical test results between the two mix groups and the control group.

\section{Results}

The delayed technique showed a significant increase in elution on day one compared with the standard mix technique $(p<0.001)$. The transition point from Phase I to Phase Il occurred on day ten. LFUS treatments significantly increased elution amounts for all groups above control. Delayed technique resulted in significantly higher elution amounts for the five-minute$(p=0.004)$ and 45 -minute- $(p<0.001)$ duration groups compared with standard technique. Additionally, the correlations between LFUS duration and total elution amount for both mix techniques were significant $(p=0.03)$. Both antibiotic-impregnated groups exhibited a significant decrease in offset yield stress compared with the control group $(p<0.001)$, however, their lower $95 \%$ confidence intervals were all above the $70 \mathrm{MPa}$ limit defined by International Standards Organization (ISO) 5833-2 reference standard for acrylic bone cement.

\section{Conclusion}

The combination of a delayed mix technique with LFUS treatments provides a reasonable means for increasing both short- and long-term antibiotic elution without affecting mechanical strength.

Cite this article: Bone Joint Res 2016;5:26-32.

Keywords: bone cement; elution; antibiotics; periprosthetic; infection; ultrasound

\section{Article focus}

- Development of a new treatment technique for persistent infections during total joint arthroplasty revisions.

- Investigate the relationship between the use of a delayed antibiotic mixing technique and low frequency ultrasound to increase long-term antibiotic elution from PMMA bone cement.

- To determine if the combination of delayed antibiotic mixing technique and low frequency ultrasound have an effect on the mechanical strength of PMMA bone cement. 


\section{Key messages}

- The combination of a delayed mixing technique and low frequency ultrasound treatment produced significantly higher antibiotic elution amounts compared with standard mixing technique.

- While both treatment combinations showed a reduction in peak yield stress compared with the control group, their values all remained above international standards for compressive strength.

\section{Strengths and limitations}

- The proposed strategy uses current techniques and readily available equipment to improve long-term antibiotic elution profiles.

- Study methods are based on standard testing protocols and previously validated measurement techniques for evaluation of antibiotic elution.

- In vitro environments, as used in this study, do not completely replicate the physiological environment of antibiotic elution and concentrations in vivo.

\section{Introduction}

Total joint arthroplasty is among the most common orthopaedic procedures performed in the United States, with over 700000 primary total knee arthroplasties (TKA) and 330000 primary total hip arthroplasties (THA) occurring in 2010. ${ }^{1}$ The prosthetic's presence incurs a lifelong infection risk, with an average infection rate for TKA and THA of 2\%.2,3 For infection occurring more than six weeks following surgery, revision arthroplasty is a common method of treatment. During these revisions, antibioticimpregnated polymethylmethacrylate (PMMA) cement is often used to anchor revision implants or to form temporary spacers used for treating the infection. Additional antibiotics are also frequently mixed into the cement to allow greater control over antibiotic type and dosage. These techniques allow for delivery of higher antibiotic concentrations locally than would be tolerated if given systemically. ${ }^{3,4}$

Unfortunately, despite using both intravenous and local antibiotic delivery methods, persistent infection rates are reported to be $5.5 \%$ to $33 \%$ for patients who undergo revision surgeries. ${ }^{5-7}$

One possible reason for the persistence of infection could be the rapidly decaying antibiotic release profile of the PMMA. The antibiotic elution is typically characterised by two phases: a preliminary high-release, short duration phase followed by a low-release, long duration phase. ${ }^{8}$ The preliminary phase, denoted as Phase I, typically lasts for a few days with relatively high elution levels. The secondary phase, denoted as Phase II, may last for weeks or even months at significantly lower elution levels. ${ }^{9-11}$ Unfortunately, the level of Phase II elution often falls below the minimum inhibitory concentration (MIC) or minimum bactericidal concentration $(\mathrm{MBC})$ allowing infectious organisms to survive and form biofilms. ${ }^{12,13} \mathrm{~A}$ higher sustained release of antibiotics during Phase II would be desirable for improving long-term treatment of persistent infections.

After the antibiotic elutes for weeks or even months, optimistic estimates put the amount of remaining antibiotic trapped in the spacer at $85 \% .{ }^{14,15}$ Previous studies have proposed techniques to increase elution amount and lengthen elution duration. These include modified mixing techniques, the use of antibiotic-loaded microspheres, poragens, or hydrogels delaying antibiotic release, as well as ultrasound treatment. ${ }^{16,17}$ However, most of these techniques appear either to target only a single elution phase or have undesirable effects on the mechanical integrity of the cement. ${ }^{18}$ This makes low frequency ultrasound (LFUS) an intriguing area of study, as several prior studies have shown that LFUS can be effective in increasing antibiotic elution. However, these previous LFUS studies either focused on Phase I elution during which antibiotic elution is already elevated, or ranged from 12 hours to 14 days of continuous treatment which is difficult to execute in a clinical setting. ${ }^{15,19}$

We therefore propose a method of combining improved mixing techniques and LFUS treatments to control both elution phases concurrently. Furthermore, we aimed to develop a more clinically relevant LFUS treatment strategy for increasing Phase II elution. We hypothesised that repeated treatments of short time durations of LFUS would increase Phase II antibiotic elution. We also hypothesised that this increase in Phase II elution would still be significant when paired with a delayed antibiotic addition mixing technique. We expected that mechanical strength of the specimens would not be significantly affected by LFUS treatments.

\section{Patients and Methods}

Specimen preparation. The specimen preparation techniques used for this study were based on established methods used for previous antibiotic elution studies conducted by our lab. ${ }^{8}$ Three batches of cement were prepared, each using a different preparation method. For the first preparation, referred to as the standard technique (manufacturer recommended technique), vancomycin was added to the dry polymer and briefly mixed before adding the liquid monomer and then mixing for 90 seconds. In the second preparation, referred to as the delayed technique, the dry polymer and monomer were mixed for 60 seconds before the addition of vancomycin, followed by 30 seconds of mixing. The control group was prepared using the standard technique without the addition of vancomycin.

Each batch was made from a 40 gram quantity of SmartSet MV (DePuy, Blackpool, United Kingdom) PMMA bone cement. Five grams of vancomycin hydrochloride powder (Sagent Pharmaceuticals Inc., Schaumburg, 
Illinois) was added to both the standard and delayed technique batches ( $12.5 \%$ by weight). All vancomycin was pre-ground using a mortar and pestle to increase specimen consistency. All mixing was done using a SmartMix cement mixing system (DePuy) under a $30 \mathrm{kPa}$ vacuum at a one $\mathrm{Hz}$ stir rate. The mixer was cleaned and sterilised with alcohol before each batch was prepared.

Forty specimens were made from each preparation batch (120 specimens made in total) using stainless steel moulds in accordance with ASTM standard F451-99a. ${ }^{20}$ This method has been shown to produce specimens that have a release profile similar to that of spacers loaded with antibiotics in vivo. ${ }^{21}$ Each batch of specimens was allowed to cure in the moulds in an incubator at $37^{\circ} \mathrm{C}$ for one hour before removal. Upon removal, the specimens were inspected for major defects and then massed using an analytical balance. Specimens showing visible defects or having a mass furthest from the group average were excluded. A total of 35 of the remaining specimens were immediately placed into individual $15 \mathrm{~mL}$ plastic vials with $10 \mathrm{~mL}$ of phosphate buffered saline (PBS) and stored in an incubator at $37^{\circ} \mathrm{C}$. A total of 105 individual specimens were included in the study.

Elution testing. The PBS solution of each vial was replaced every 24 hours for the duration of the study. Before each solution change, $200 \mathrm{uL}$ samples were taken from each specimen vial and were analysed using a flow injection analysis (FIA) technique with a Shimadzu SPD-10AV ultraviolet detector (Shimadzu, Kyoto, Japan) set at a $214 \mathrm{~nm}$ detection wavelength to determine the vancomycin concentration. FIA has previously been used as a simple and accurate way to evaluate antibiotic concentrations. ${ }^{22,23}$

Low frequency ultrasound treatment. Of the 35 specimens taken from each cement preparation batch, 30 were chosen at random and divided into four LFUS duration groups. LFUS durations of five, 15, and 45 minutes were tested with eight specimens each, plus a control group of six specimens which received no LFUS treatment. The LFUS treatments were performed by submerging the vials in a $25.5 \mathrm{kHz}$ ultrasonic water bath (Blackstone Advanced Technologies, LLC, Jamestown, New York) set at $22^{\circ} \mathrm{C}$ for each LFUS group's respective duration time. The water bath temperature was monitored before and after each treatment. The transition point from Phase I to Phase II was defined as the day when there was no significant change in elution concentration from the previous day. LFUS treatments were started on the first day of Phase II and were conducted two additional times with one day between each treatment. The treatments were done immediately after the PBS solution changes on those days. Amount of elution was defined as the elution amount seen at the following day's sample collection minus the corresponding LFUS control group's elution amount for the same day.
Compression testing. All specimens in the LFUS groups were mechanically tested in compression at the end of the elution period. The tests were performed using an 858 Mini Bionix II load frame (MTS Systems, Eden Prairie, Minnesota) at a loading rate of $22 \mathrm{~mm} / \mathrm{min}$ per ASTM F451-99a. ${ }^{20}$ The five remaining specimens were mechanically tested after the first 24 hours of the elution period. Average and standard deviation values for offset yield strength and stiffness were calculated for each group. The results were compared to International Standards Organization (ISO) 5833:2002 which specifies a minimum threshold below which bone cement is considered too weak for many orthopaedic applications. ${ }^{24}$

Statistical analysis. A paired t-test with a significance p-value of $<0.05$ was used to compare the elution amounts on consecutive days in order to determine the starting day for Phase II elution. Paired, two-tailed t-tests were used to compare total vancomycin elution using the standard technique with that resulting from the delayed mix technique, as well as to compare LFUS elution amounts with the control group. Individual univariate ANOVAs were used to compare elution amounts between LFUS duration groups and mixing techniques, and to identify any significant cross effects. Pearson correlation tests were used to measure trends in LFUS duration and total elution amounts. Paired, two-tailed $t$-tests and univariate ANOVAs were used to compare the mechanical test results. The statistical analysis was performed using Excel (Microsoft Corporation, Redmond, Washington) and SPSS (IBM Corporation, Armonk, New York) software.

\section{Results}

In comparing the two mixing techniques, the delayed technique showed a significant increase in elution for day one compared with the normal technique ( $p<0.001)$, however, all following days were not significantly different until the LFUS trials (Fig. 1). These findings are in agreement with previously published works. ${ }^{8}$

The transition point from Phase I to Phase II occurred on day ten at which point the ultrasound treatments began; treatments were undertaken on days ten, 12 , and 14. The total antibiotic elution from the LFUS groups, shown in Figure 2, were all significantly higher compared with the non-LFUS control groups (all $p<0.001$ ). Delayed technique resulted in significantly higher elution amounts for the $5 \min (p=0.004)$ and $45 \min (p<0.001)$ duration groups compared with standard technique. Additionally, the correlations between LFUS duration and total elution amount for both mix techniques were significant $(p=0.03)$.

In comparing daily elution amounts for combined LFUS durations (Fig. 3), there were significant increases in elution amount for both standard and delayed techniques on the first two LFUS treatment days (ten and 12) compared with the non-LFUS groups (all $p<0.03$ ). The 


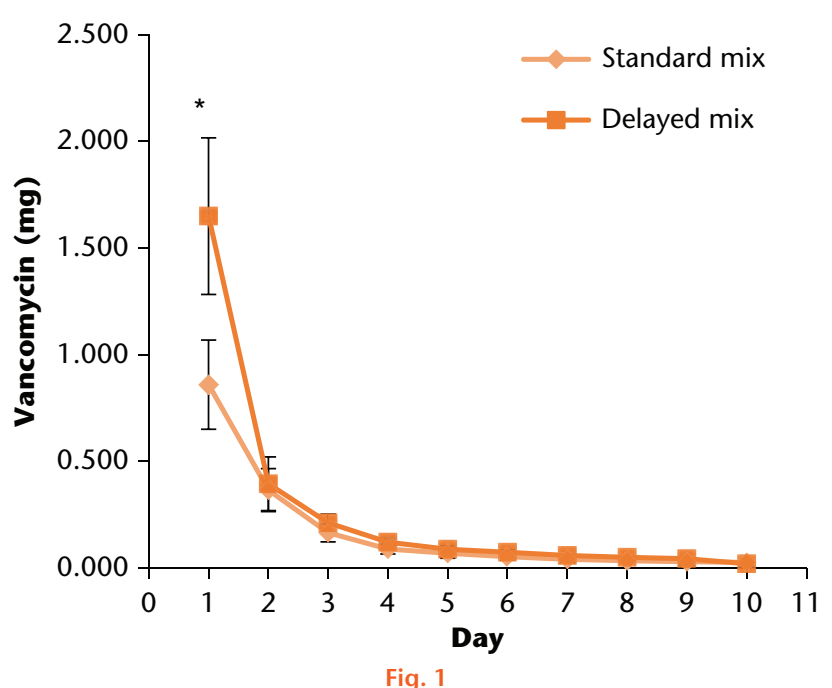

Comparison of average daily elution profiles for normal and delayed mixing techniques during Phase I. Error bars indicate $+/$ - one standard deviation. *t-test significance of $p<0.001$

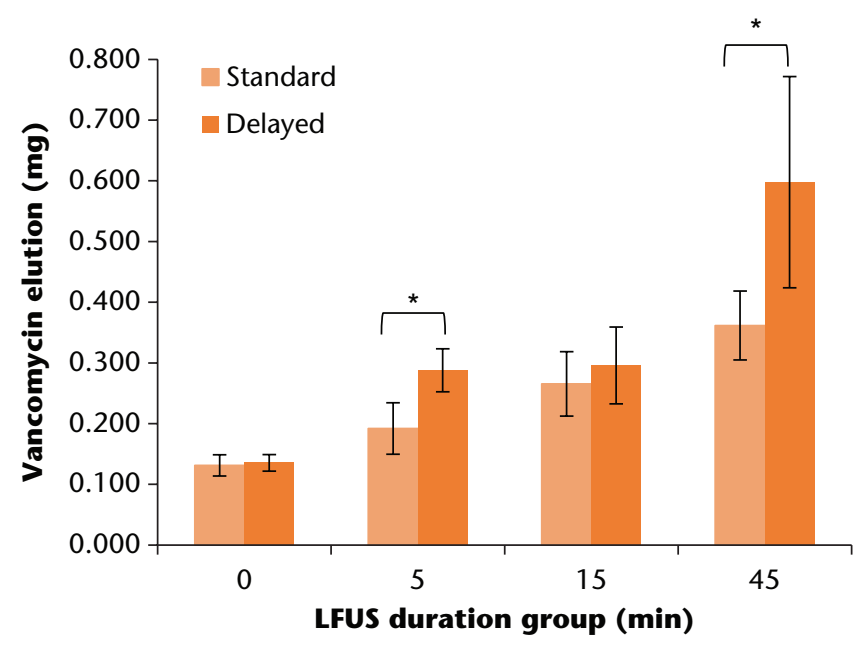

Fig. 2

Total vancomycin elution for each LFUS duration group. Error bars indicate +/- one standard deviation. All LFUS duration groups were significantly higher than the LFUS control group (all t-test $p<0.001$ ).

${ }^{*} t$-test significance of $\mathrm{p}<0.004$

delayed technique was significantly higher on days ten $(p<0.001)$ and $12(p=0.029)$.

It should be noted that temperature increased by $1^{\circ}$, $5^{\circ}$, and $12^{\circ} \mathrm{C}$ for LFUS groups five, 15 , and $45 \mathrm{~min}$, respectively, however, none of these increases rose above the standard incubation temperature of $37^{\circ} \mathrm{C}$.

Mechanical test results. Both antibiotic impregnated groups exhibited a significant decrease in offset yield stress compared with the control group ( $p<0.001)$. However, their lower 95\% confidence intervals were all above the $70 \mathrm{MPa}$ limit defined by ISO 5833:2002.24 There was no significant difference in offset yield stress between LFUS durations, shown in Figure 4, for both mixing techniques ( $p$-value $>0.3$ ).
The standard mixing technique resulted in a significant reduction in mechanical stiffness for the 45 min LFUS duration group compared with the control group $(p=0.02)$. The delayed mixing technique resulted in a significant reduction in stiffness across all LFUS durations compared with the respective control groups (all $p<0.03$ ). There was a significant difference between standard and delayed group stiffness for the $0 \mathrm{~min}$ $(p=0.03)$ and $45 \mathrm{~min}(p=0.015)$ LFUS duration groups. Average stiffness values are shown in Figure 5.

In comparing LFUS duration group values with the 24-hour soak group, there were no significant differences for offset yield (all $p>0.1$ ). However, there was a significant difference in stiffness between the control and delayed mix groups $(p=0.047)$.

\section{Discussion}

The results presented in this study show a clear trend that the application of LFUS to antibiotic-impregnated PMMA cement does increase Phase II elution. The strong correlation between higher elution amounts and increasing LFUS duration indicates that this method may allow for direct control over Phase II elution. In theory, both elution time and amount could be controlled, however, they are probably not completely independent of each other.

We designed this study hoping to develop a more clinically relevant method for increasing antibiotic elution. The findings indicate that it is possible to combine these two elution enhancement techniques and use them in tandem to improve elution in both phases. Given that the delayed mixing technique results in higher elution levels in both elution phases, it may be reasonable to assume that the delayed technique would result in a lower remaining amount of embedded vancomycin. This could then suggest that there would be less vancomycin available to be freed by LFUS treatment compared to the standard mixing technique and thus result in a shortened period over which LFUS would remain effective. However, our results do not support this hypothesis as both mixing techniques showed elution levels to diminish at approximately the same rate. One possible reason for this is that the LFUS treatments may not have been long enough in duration or at a high enough intensity. Exact clinical implementation of such a treatment would depend on the type of revision surgery performed. The general treatment scheme could include a prescribed number of visits by the patient to an outpatient clinic where a clinician provides the treatments utilising currently available LFUS equipment.

A potential approach designed to eradicate infection might include controlling Phase I elution by choosing an ideal antibiotic dose and adding it at a specific time point during the mixing process (using a delayed mixing technique), then maximising Phase II elution with the appropriate timing and treatment duration of LFUS trials. 


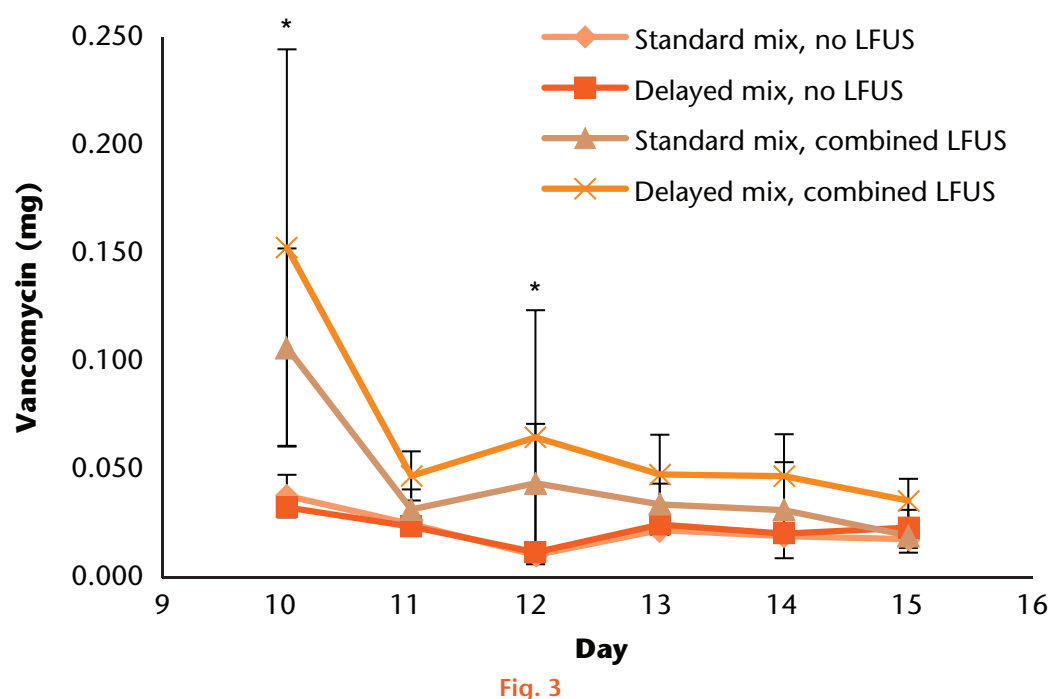

Comparison of total daily antibiotic elution amounts for standard and delayed mix techniques with and without LFUS treatments. LFUS treatments were undertaken on days ten, 12, and 14. Error bars indicate +/- one standard deviation.

* Indicates a significant paired t-test difference in elution amount compared with no LFUS group and a significant difference between standard and delayed mix elution

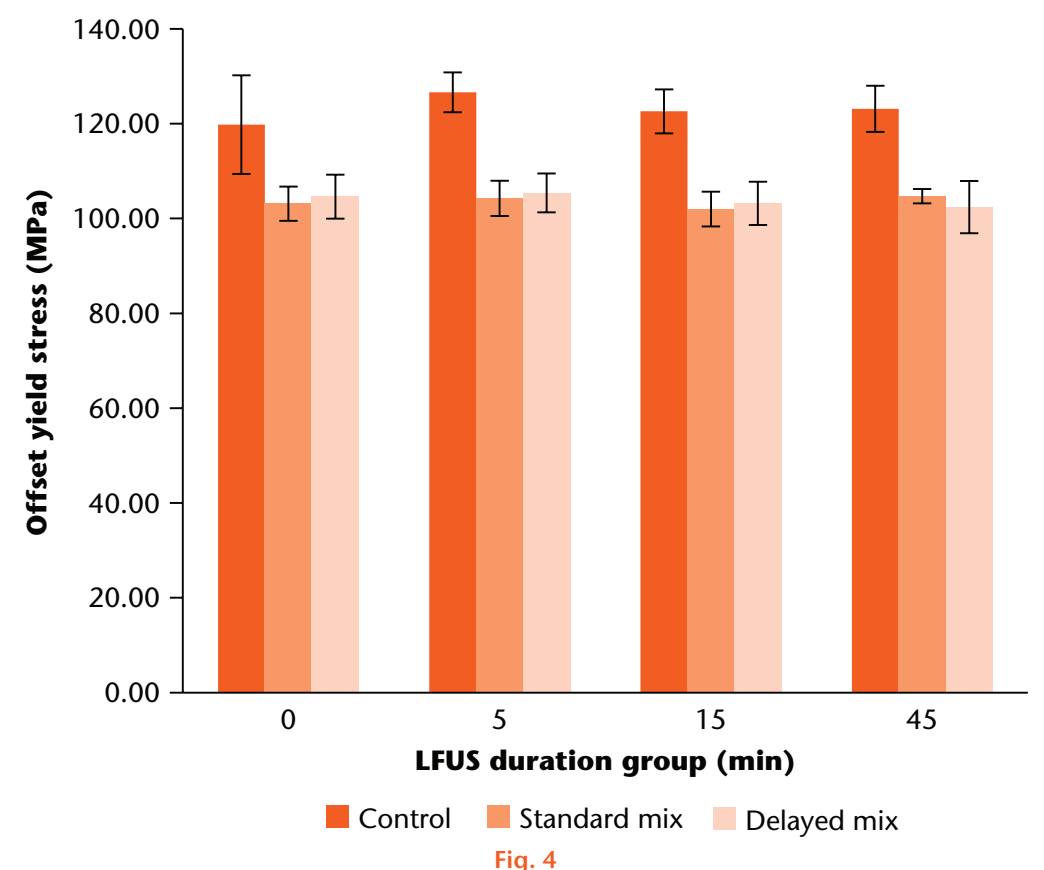

Comparison of average offset yield stresses for the three mix groups. Error bars indicate $+/$ - one standard deviation for each respective average. Both standard and delayed mix amounts were significantly lower than control across all LFUS duration groups using paired t-tests.

A single high-power, high-duration treatment could provide a single Phase II elevated release peak that could be on the order of Phase I elution amounts for a short period of time. This type of LFUS treatment could be useful for biofilm disruption or when using an antibiotic that exhibits concentration-dependent bacteriocidal properties. ${ }^{25}$ Alternatively, consecutive low-power, short-duration treatments could be used over a series of several days or even weeks to provide a lower but more consistent elevated level of Phase II elution. This type of LFUS treatment could potentially be beneficial for raising Phase II elution to levels above MIC or MBC for antibiotics that exhibit time-dependent antibacterial properties.

The mechanical testing results indicate that the addition of vancomycin to the cement has a greater effect on the cement's mechanical properties than do the specific mixing technique or LFUS duration. Previous studies have shown that there is a limit to how much vancomycin should be added before mechanical properties are degraded. ${ }^{26,27}$ While five grams is on the high side of what has been shown to be acceptable, for the purpose of this study the amount was chosen to help increase 


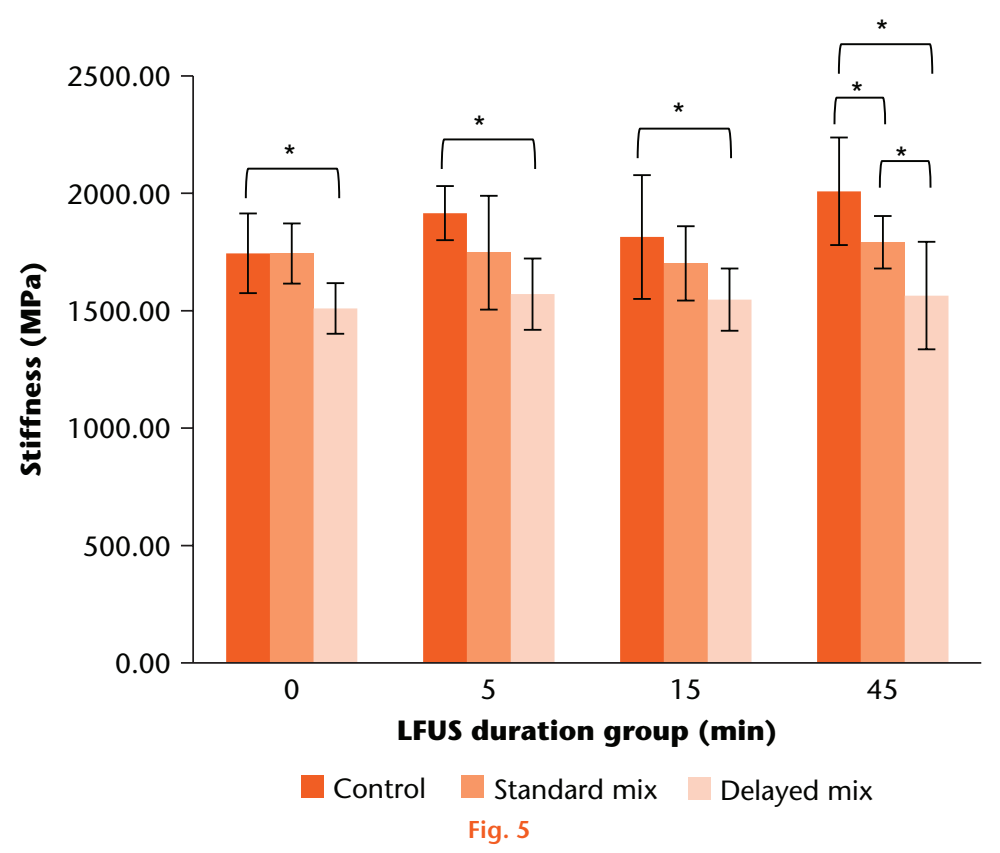

Comparison of mechanical stiffness among mix groups. Error bars indicate $+/$ - one standard deviation for each respective average. *paired t-test significance of $p<0.05$

elution amount for greater measurement sensitivity. The use of a varied mixing technique and Phase II LFUS treatments coupled with the addition of the antibiotic did not compromise its integrity beyond the referenced ISO standard for peak stress. While there was a reduction in stiffness for the delayed technique compared with the standard technique, the importance of this effect would be dependent on the particular clinical application. Direct weight-bearing cases may be more sensitive to this reduction in stiffness while non-weight bearing ones may benefit more from the potential increases in antibiotic elution being afforded by the delayed technique. Future studies will be necessary to evaluate the effect of combined mixing and LFUS techniques on mechanical properties of clinically relevant cement spacers.

Several assumptions and simplifications were used for this study. First, the elution environment in which the specimens were kept was not ideal for modeling in vivo behaviour. The results are, however, in agreement with previously published works that used similar environmental assumptions. ${ }^{15,28,29}$ True characterisation of LFUS effectiveness in vivo should include measures of spacer shape, thickness, and surrounding soft tissues. However, the goal of this study was not to prove the effectiveness of LFUS on its own, which has been previously shown, but rather to investigate the effect of combining it with modified preparation techniques. ${ }^{30}$ Temperature of the elution environment also likely has some effect on total elution. While the device used for this study did not provide a highly accurate means for controlling the temperature of the LFUS bath during each treatment, the temperature ranges seen were similar to previous studies. ${ }^{31}$
In the treatment of infected prosthetics, MIC or MBC is often used as the reference point which antibiotic concentrations are expected to surpass in order to eradicate the infection. As with the various parameters that affect the antibiotic elution itself, the precise MIC or MBC level for a given infection is completely patient- and sitedependent. This implies that there is not a relevant concentration reference for a controlled, idealised study such as the work presented here. The primary reference used in this study was the LFUS control groups which received no LFUS treatments. While the results did produce significant increases above this point, this does not imply concentrations above (or below) MIC or MBC. Future in vivo animal studies are planned to investigate LFUS elution models referenced to true bacterial MIC or MBC in order to validate antibiotic effectiveness.

While only three repeated trials of LFUS were used in this study, there is likely a limit to how much antibiotic can be eluted by repeat treatments. The results did show a significant decrease in elution amount on the third set of LFUS trials, indicating a reduction in remaining antibiotic with increasing number of treatments. Future studies are planned to investigate methods which maintain elution rates above a targeted level using various combinations of frequency, power, and duration.

The results of this study have shown that a delayed mixing technique used in conjunction with LFUS treatments shows promise as a means for antibiotic elution control in both elution phases. These findings suggest that the proposed combined treatment approach could be a reasonable and effective means of increasing antibiotic elution to help clear persistent periprosthetic infections. 


\section{References}

1. National Hospital Discharge Survey 2010. ftp://ftp.cdc.gov/pub/Health Statistics/NCHS/Dataset_Documentation/NHDS/NHDS_2010_Documentation.pdf (date last accessed 23 October 2015).

2. Ponce B, Raines BT, Reed RD, et al. Surgical Site Infection After Arthroplasty: Comparative Effectiveness of Prophylactic Antibiotics: Do Surgical Care Improvement Project Guidelines Need to Be Updated? J Bone Joint Surg [Am]2014;96-A:970-977.

3. Zimmerli W, Trampuz A, Ochsner PE. Prosthetic-joint infections. N Engl J Med 2004;351:1645-1654.

4. Wininger DA, Fass RJ. Antibiotic-impregnated cement and beads for orthopedic infections. Antimicrob Agents Chemother 1996;40:2675-2679.

5. Wolf M, Clar H, Friesenbichler J, et al. Prosthetic joint infection following total hip replacement: results of one-stage versus two-stage exchange. Int Orthop 2014;38:1363-1368.

6. Mortazavi SM, Vegari D, Ho A, Zmistowski B, Parvizi J. Two-stage exchange arthroplasty for infected total knee arthroplasty: predictors of failure. Clin Orthop Relat Res 2011:469:3049-3054.

7. Shanmugasundaram S, Ricciardi BF, Briggs TW, Sussmann PS, Bostrom MP. Evaluation and Management of Periprosthetic Joint Infection-an International Multicenter Study. HSSJ 2014;10:36-44.

8. Amin TJ, Lamping JW, Hendricks KJ, Mclff TE. Increasing the elution of vancomycin from high-dose antibiotic-loaded bone cement: a novel preparation technique. J Bone Joint Surg [Am]2012;94-A:1946-1951.

9. Grimsrud C, Raven R, Fothergill AW, Kim HT. The in vitro elution characteristics of antifungal-loaded PMMA bone cement and calcium sulfate bone substitute. Orthopedics 2011;34:e378-e381.

10. Anagnostakos K, Schmid NV, Kelm J, Grün U, Jung J. Classification of hip joint infections. Int J Med Sci2009;6:227-233.

11. Hendriks JG, van Horn JR, van der Mei HC, Busscher HJ. Backgrounds of antibiotic-loaded bone cement and prosthesis-related infection. Biomaterials 2004;25:545-556.

12. Bertazzoni Minelli E, Della Bora T, Benini A. Different microbial biofilm formation on polymethylmethacrylate (PMMA) bone cement loaded with gentamicin and vancomycin. Anaerobe 2011;17:380-383.

13. Li W, Ding Y, Rai $\mathbf{R}$, et al. Preparation and characterization of PHBV microsphere/45S5 bioactive glass composite scaffolds with vancomycin releasing function. Mater Sci Eng C Mater Biol App/ 2014;41:320-328.

14. Kuechle DK, Landon GC, Musher DM, Noble PC. Elution of vancomycin, daptomycin, and amikacin from acrylic bone cement. Clin Orthop Relat Res 1991;264:302-308.

15. Ensing GT, Hendriks JG, Jongsma JE, et al. The influence of ultrasound on the release of gentamicin from antibiotic-loaded acrylic beads and bone cements. J Biomed Mater Res B Appl Biomater 2005;75:1-5.

16. Shi M, Kretlow JD, Spicer PP, et al. Antibiotic-releasing porous polymethylmethacrylate/gelatin/antibiotic constructs for craniofacial tissue engineering. J Control Release 2011;152:196-205.

17. Cai XZ, Yan SG, Wu HB, et al. Effect of delayed pulsed-wave ultrasound on local pharmacokinetics and pharmacodynamics of vancomycin-loaded acrylic bone cement in vivo. Antimicrob Agents Chemother 2007;51:3199-3204.

18. Nugent M, McLaren A, Vernon B, McLemore R. Strength of antimicrobial bone cement decreases with increased poragen fraction. Clin Orthop Relat Res 2010;468:2101-2106.

19. Penner MJ, Masri BA, Duncan CP. Elution characteristics of vancomycin and tobramycin combined in acrylic bone-cement. J Arthroplasty 1996;11:939-944.
20. No authors listed. ASTM F451-99a(2007)e1. Standard Specification for Acrylic Bone Cement. ASTM International West Conshohocken, PA, 2007, DOI: 10.1520/F045199AR07E01, www.astm.org

21. Marra F, Robbins GM, Masri BA, et al. Amphotericin B-loaded bone cement to treat osteomyelitis caused by Candida albicans. Can J Surg 2001;44:383-386.

22. Khataee AR, Hasanzadeh A, Iranifam M, et al. CuO nanosheets-enhanced flowinjection chemiluminescence system for determination of vancomycin in water, pharmaceutical and human serum. Spectrochim Acta A Mol Biomol Spectrosc 2014;122:737-743.

23. Enrique M, García-Montoya E, Miñarro M, et al. Application of an experimental design for the optimization and validation of a new HPLC method for the determination of vancomycin in an extemporaneous ophthalmic solution. J Chromatogr Sci 2008;46:828-834.

24. No authors listed. ISO 5833:2002. Implants for surgery - Acrylic resin cements 2013. International Organization for Standardization, Geneva, Switzerland, TC/ SC: ISO/TC 150/SC 1, ICS: 11.040.40, www.iso.org (date last accessed 20 January 2016).

25. Tzeng A, Tzeng TH, Vasdev S, et al. Treating periprosthetic joint infections as biofilms: key diagnosis and management strategies. Diagn Microbiol Infect Dis 2015:81:192-200.

26. Lilikakis A, Sutcliffe MP. The effect of vancomycin addition to the compression strength of antibiotic-loaded bone cements. Int Orthop 2009;33:815-819.

27. Brock HS, Moodie PG, Hendricks KJ, Mclff TE. Compression strength and porosity of single-antibiotic cement vacuum-mixed with vancomycin. J Arthroplasty 2010;25:990-997

28. Shi M, Kretlow JD, Nguyen A, et al. Antibiotic-releasing porous polymethylmethacrylate constructs for osseous space maintenance and infection control. Biomaterials 2010;31:4146-4156.

29. Urabe $\mathbf{K}$, Naruse $\mathbf{K}$, Hattori $\mathbf{H}$, et al. In vitro comparison of elution characteristics of vancomycin from calcium phosphate cement and polymethylmethacrylate. J Orthop Sci2009; 14:784-793.

30. Cai XZ, Chen XZ, Yan SG, et al. Intermittent watt-level ultrasonication facilitates vancomycin release from therapeutic acrylic bone cement. J Biomed Mater Res B Appl Biomater 2009;90:11-17.

31. Brooks AT, Nelson CL, Stewart CL, Skinner RA, Siems ML. Effect of an ultrasonic device on temperatures generated in bone and on bone-cement structure. J Arthroplasty 1993;8:413-418.

Funding Statement

This work was partially supported by the Marc A. and Elinor J. Asher Orthopedic Research Endowment.

Author Contribution

A. Wendling, Experimental design, data collection, data analysis, literature review, writing the paper.

D. Mar, Experimental design, data collection, data analysis, writing the paper.

- N. Wischmeier, Clinical guidance, writing and editing paper.

D. Anderson, Clinical guidance, writing and editing paper.

- T. Mclff, Experimental design, writing and editing paper.

ICMJE conflict of interest

- None declared.

(c) 2016 Wendling et al. This is an open-access article distributed under the terms of the Creative Commons Attributions licence (CC-BY-NC), which permits unrestricted use, distribution, and reproduction in any medium, but not for commercial gain, provided the original author and source are credited. 